20 Introduction - In CMP, a chemically-rich slurry containing abrasive nanoparticles is first

21 delivered to the pad surface and allowed to form a thin film across the surface of the

22 rotating polishing pad. A patterned wafer, having some gross topography, is then inserted

23 within a carrier and held in place by a plastic retaining ring containing slots on its periphery

\section{Tribology of Retaining Rings in Chemical Mechanical Planarization}

Gabriela Diaz a ${ }^{\text {a }}$ Lauren Peckler ${ }^{\text {a }}$, Yasa Sampurno ${ }^{\text {a,b }}$ and Ara Philipossian ${ }^{\text {a,b }}$ a Department of Chemical and Environmental Engineering, University of Arizona, Tucson, Arizona 85721 USA

${ }^{\mathrm{b}}$ Araca Incorporated, Tucson, Arizona 85718 USA

Stribeck and Stribeck+ curves helped determine the tribological mechanisms in the ring-slurry-pad interface. Both methods gave consistent results with the lubrication mechanism starting at "boundary lubrication" and transitioning to "mixed lubrication" as pseudo-Sommerfeld numbers increased. COF for PPS rings were higher than PEEK. They were also higher for inter-level dielectric (ILD) processes as compared to copper. Stribeck curves were used to infer wear rate information about the ring, which in conjunction with Stribeck+ curves could help choose process parameters that balanced wafer removal with ring wear. Data cluster shapes were shown to be due to shear and normal force fluctuations. 
24 for slurry access into the pad-wafer interface. The wafer and retaining ring assembly are

25 then pressed face down against the pad which itself contains many surface asperities as

26 well as deep grooves. Over time, a combination of chemical and mechanical effects remove

27 the "up-features" of the wafer thereby resulting in local and global planarization of the 28 workpiece.

30 One of the more effective methods for understanding CMP fundamentals, especially when

31 it comes to the mechanical effects involved, is through tribological studies where the

32 objective is to exploit frictional forces to remove a certain amount of material from the

33 wafer while ensuring that the same forces are low enough to extend the useful life of the

34 ring as well as the pad. Our research team has successfully shown the utility of traditional

35 Stribeck curves in determining the lubrication mechanisms involved in the pad-slurry-

36 wafer interface in CMP [1-3]. In our recent works, [4-6] we have also introduced a new

37 method for determining an "improved" Stribeck curve (which we have called the

38 "Stribeck+ curve") that has yielded more useful tribological information while

39 dramatically reducing consumables as well as experimental time compared to the

40 traditional means. The key point that we have emphasized in our work is that the Stribeck+

41 curve does not assume normal force to be constant throughout the polishing process as we

42 can measure it, as well as the shear force, instantaneously.

44 We believe that it would be highly beneficial if we could determine whether the utility of

45 the Stribeck+ curve can be extended to retaining rings. This would help process engineers,

46 tool makers and consumables suppliers to better understand the tribology within a given 
47 set of consumables, at certain process conditions, and aim to process robustness as well as

48 increase ring life without compromising wafer removal rate. Here, we set out to

49 tribologically test identically shaped broken-in PPS and PEEK rings having 18 equidistant

50 peripheral slots (3-mm in width and depth), using copper and ILD slurries at various

51 pressures and velocities to determine whether we can attain the above objectives.

53 Experimental Apparatus and Procedure - All tests were done on an Araca APD-800

54 polisher and tribometer equipped with proprietary force transducers suitable for acquiring

55 real-time shear and normal force at high (i.e. up to 1,600 Hz) frequencies [7]. Attached to

56 the carrier head (needed to polish our $300 \mathrm{~mm}$ wafers) was an interface template with a

57 retaining ring and backing film assembly. To isolate the COF in the ring-slurry-pad

58 interface, the polishing process was performed without a wafer. Prior to polishing and data

59 acquisition, a new CMC D100 concentrically grooved pad was broken in for 30 mins with

60 deionized water using a 3M A165 diamond disc at a normal load of $44.5 \mathrm{~N}$, a rotation rate

61 of $95 \mathrm{RPM}$ and a sweep frequency of $0.17 \mathrm{~Hz}$. The same recipe was used for the in-situ

62 conditioning during ring polishing. The ILD and copper slurries employed were Fujimi

63 PL-4217 and PL-7106, respectively. Both slurries contained silica-based nano-particles

64 and were pre-diluted as per the manufacturer's specifications. In all cases, the slurry

65 flowrate was $200 \mathrm{ml}$ per min. For generating the Stribeck+ curves, we performed only one

66 3-minute polishing run with a multitude of gradual yet continuous changes (e.g. time-

67 dependent ramp-ups and ramp-downs) in polishing pressure and sliding velocity. During

68 each 3-minute run, normal forces and shear forces were instantaneously measured at 1,000

69 Hz. Table I shows the conditions for each step involved in constructing the Stribeck+ curve. 
70 Each ramp-up or ramp-down step took approximately 2 seconds. The same pressure and

71 velocity combinations were used to carry out the discrete runs needed to construct each

72 Stribeck curve.

74 Results and Discussions - Fig. 1 shows the traditional Stribeck curves (circular symbols)

75 corresponding to the PPS ring using both copper (red) and ILD (green) slurries. In each

76 case, the 5 data points represent average COF values obtained from individual 2-minute

77 polishes at a given pressure-velocity combination as per Table 1. Fig. 1 also shows the

78 Stribeck+ curves (dots) corresponding to the same polishing conditions. For purposes of

79 comparison, the 5 data points are superimposed on the 180,000 Stribeck + data points

80 obtained by a single run through multiple pressures, velocities and slurries (copper slurry

81 in orange, and ILD slurry in black). For the copper slurry, the traditional Stribeck curve

82 starts off in "boundary lubrication" with an average COF of about 0.44 , and then begins to

83 slightly transition into "mixed lubrication" half-way through where average COF drops to

84 0.36. The same trend is evident for the ILD slurry with 0.65 as the starting average COF

85 value which then drops to 0.50 at higher pseudo-Sommerfeld numbers. It is clear that, with

86 a single run, Fig. 1 is able to capture all of the trends seen in the individual Stribeck curve

87 data points, and also provide additional valuable information as follows:

89 - The Stribeck+ curve, being continuous in nature, indicates that anomalous

90 tribological behavior does not exist in between discrete polishing conditions.

91 - A fascinating commonality becomes evident in the evolution of the shape of data

92 clusters as a function of pseudo-Sommerfeld number which is not just a visual

93 artifact due to the log-log nature of the graph. Here, the ratio of measured variances 
in shear force to those of normal force depend strongly on the pseudo-Sommerfeld number. We believe this to be a fundamental aspect of CMP that has so far been overlooked by the CMP community. We plan to report on it soon.

- As seen in the inset diagram in Fig. 1, the data points in each Stribeck+ curve can be replotted with the $\mathrm{y}$-axis representing $\mathrm{COF} \times \mathrm{P} \times \mathrm{v}$ which we can assume to be proportional to the instantaneous wear rate of the retaining ring from a purely mechanical standpoint [8]. The notion of obtaining an indicator for instantaneous wear rate (or removal rate) from a single 3-min run can be quite powerful and revolutionary. This is a new idea and one that will require further study to be confirmed. Of course, in CMP, a particular pressure and velocity combination gets selected to cause a desired removal and planarization rate on the wafer surface, and not necessarily to reduce ring wear. However, given the fact that, in high-volume manufacturing, the pressure applied to a product wafer is always lower by 1 or 2 PSI than the ring pressure, we believe that our new way of combining the tribological and implied ring wear (or wafer removal) rate plots can help process engineers select desired operating conditions based on their main sets of objectives

112 By way of another example, we performed tests at the same conditions using a retaining 113 ring made of a different material (polyether ether ketone, PEEK) which had the same size 114 and geometry as that of the PPS ring. Over the years, PEEK has emerged as an alternative 115 to PPS for constructing retaining rings. This is due to various reasons such as less shedding, 116 higher wear-resistance, lower COF and lower operating temperature during CMP. Fig. 2, 
117 confirms that similar trends exist in the case of the PEEK ring when it comes to Stribeck

118 and Stribeck+ curves, as well as the implied kinetic curve shown in the inset diagram. In

119 Fig. 2, it must be noted that, for a yet to be determined reason, the superimposition does

120 not work as well as that in Fig. 1 as the individual runs associated with the Stribeck curve

121 of Fig. 2 are not always centered inside the respective data clusters of the Stribeck+ curves.

123 Both figures indicate that COF values for the PPS ring are on average 21 percent higher

124 than PEEK. The higher values are consistent with earlier findings by other researchers [9].

125 Average COF values are also higher by 55 percent for ILD processes as compared to copper.

126 This is likely due to the higher abrasive content of the ILD slurry (10\% by weight) as

127 compared to less than $1 \%$ for the copper slurry. Average pad surface temperature for the

128 PPS ring is 9 to $11^{\circ} \mathrm{C}$ higher than that of PEEK. Also, in all cases, pad temperature is

129 higher by 2 to $3{ }^{\circ} \mathrm{C}$ when the ILD slurry is used as compared to the copper slurry. These

130 temperature differences are quite consistent with, and a result of, their corresponding COF

131 values.

132

133 Conclusions - We utilized Stribeck and Stribeck+ curves to tribologically characterize

134 PEEK and PPS retaining rings for copper and ILD CMP. Both methods led us to the same

135 set of conclusions: for a given slurry type COF values for PPS were always higher than for

136 PEEK, and the same relationship held for ILD processes as compared to copper. Pad

137 temperature measurements also corroborated the above finding. Stribeck+ curves were

138 used to construct new kinetic curves which inferred wear rate information about the ring.

139 This information, when used in conjunction with Stribeck+ curves can help identify process 
140 parameters that balance wafer removal rate with ring wear rate at a given tribological state.

141 In all cases, a fascinating commonality was seen in the evolution of the shape of data

142 clusters as a function of pseudo-Sommerfeld number indicating the presence of a complex

143 interplay between variances in normal and shear force which will be a subject of our next 144 study.

146 References

148 1. Y. A. Sampurno, L. Borucki, Y. Zhuang, S. Misra \& A. Philipossian. Thin Solid $149 \quad$ Films, 517(5), 1719-1726 (2009).

150 2. Y. Jiao, Y. Sampurno, Y, Zhuang, X. Wei, A. Meled \& A. Philipossian. Japanese 151 Journal of Applied Physics, 50, 05EC02 (2011)

152 3. Y. Mu, Y. Jiao, Y. Sampurno, Y. Zhuang, S. Theng \& A. Philipossian. Japanese $153 \quad$ Journal of Applied Physics, 54(7), 076502 (2015).

154 4. R. Han, Y. Sampurno, S. Theng, F. Sudargho, Y. Zhuang \& A. Philipossian. ECS 155 Journal of Solid State Science and Technology, 6, 32 (2017).

156 5. M. Bahr, Y. Sampurno, R. Han \& A. Philipossian. ECS Journal of Solid State 157 Science and Technology, 6 (5) P290-295 (2017).

158 6. R. Han, Y. Sampurno, S. Theng, F. Sudargho, Y. Zhuang \& A. Philipossian. ECS 159 Journal of Solid State Science and Technology, 6 (4) P161-164 (2017).

160 7. http://www.aracainc.com/media/pubs/Polisher_and_Tribometer_300mm.pdf.

161 8. F.W. Preston, J. Soc. Glass Technology, 11, 277 (1927). 
9. N. Gitis, J. Xiao, A. Kumar \& A. Sikder, in the Proceedings of Ninth CMP-MIC Conference, Institute for Microelectronics on Chip Inter Connection, p. 252 (2004). 
165

\begin{tabular}{cccc}
\hline $\begin{array}{c}\text { Stribeck+ Step } \\
\text { No. }\end{array}$ & $\begin{array}{c}\text { Duration of the } \\
\text { Stribeck+ Step } \\
\text { (sec) }\end{array}$ & Pressure (PSI) & $\begin{array}{c}\text { Sliding Velocity } \\
\text { (m/s) }\end{array}$ \\
\hline 1 & 60 & 2 & 1.2 \\
2 & 30 & 2 & 1.8 \\
3 & 30 & 4 & 1.8 \\
4 & 30 & 6 & 1.2 \\
5 & 30 & 6 & 1.8 \\
\hline
\end{tabular}

166

167 


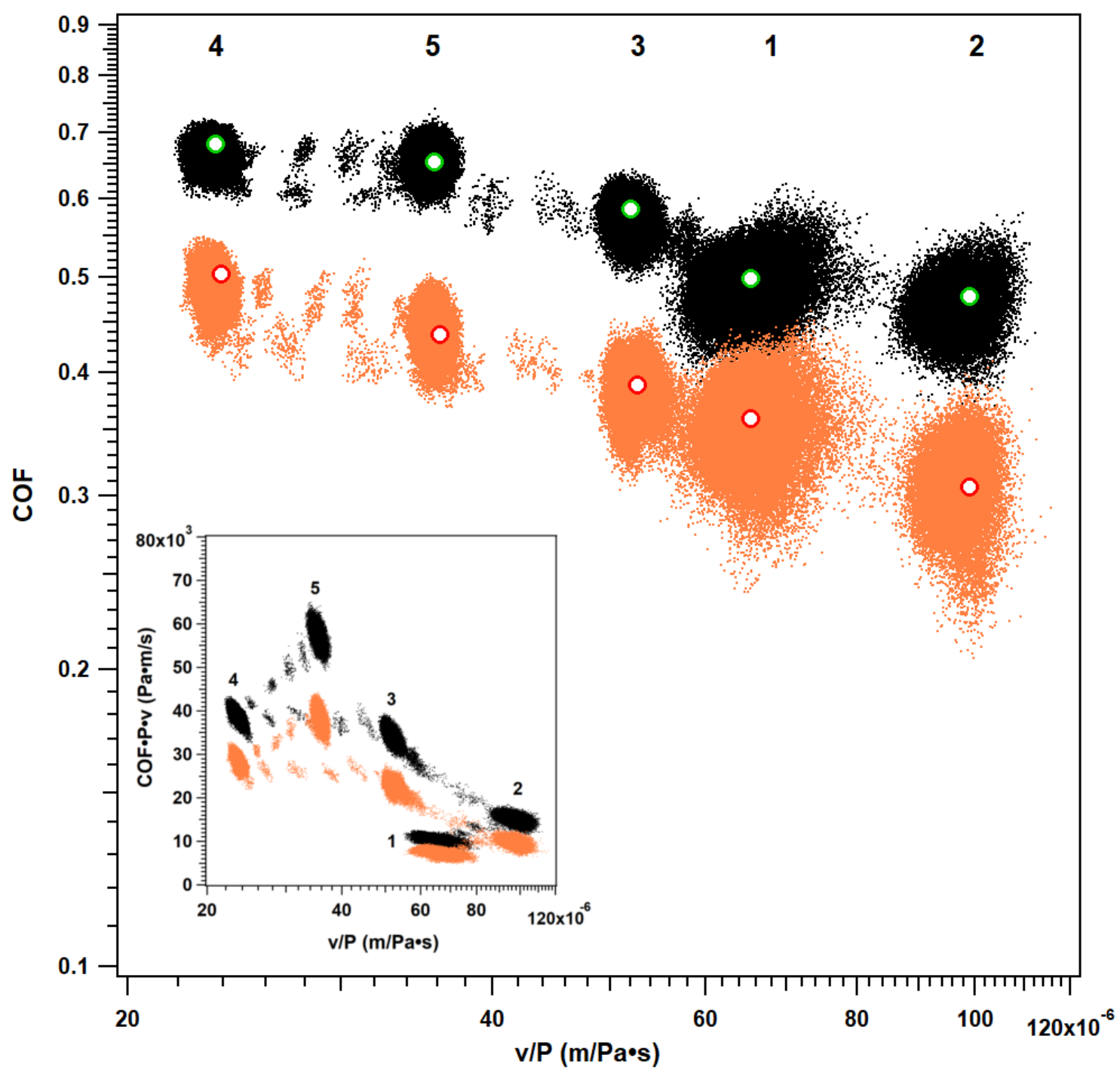

Fig 1. Stribeck (open circles) and Stribeck+ (clusters) curves corresponding to the PPS ring using PL-4217 (black) and PL-7106 (orange). Numbers correspond to step numbers in Table 1. Inset: Implied ring wear rate as a function of pseudo-Sommerfeld number for PL-4217 (black) and PL-7106 (orange). 


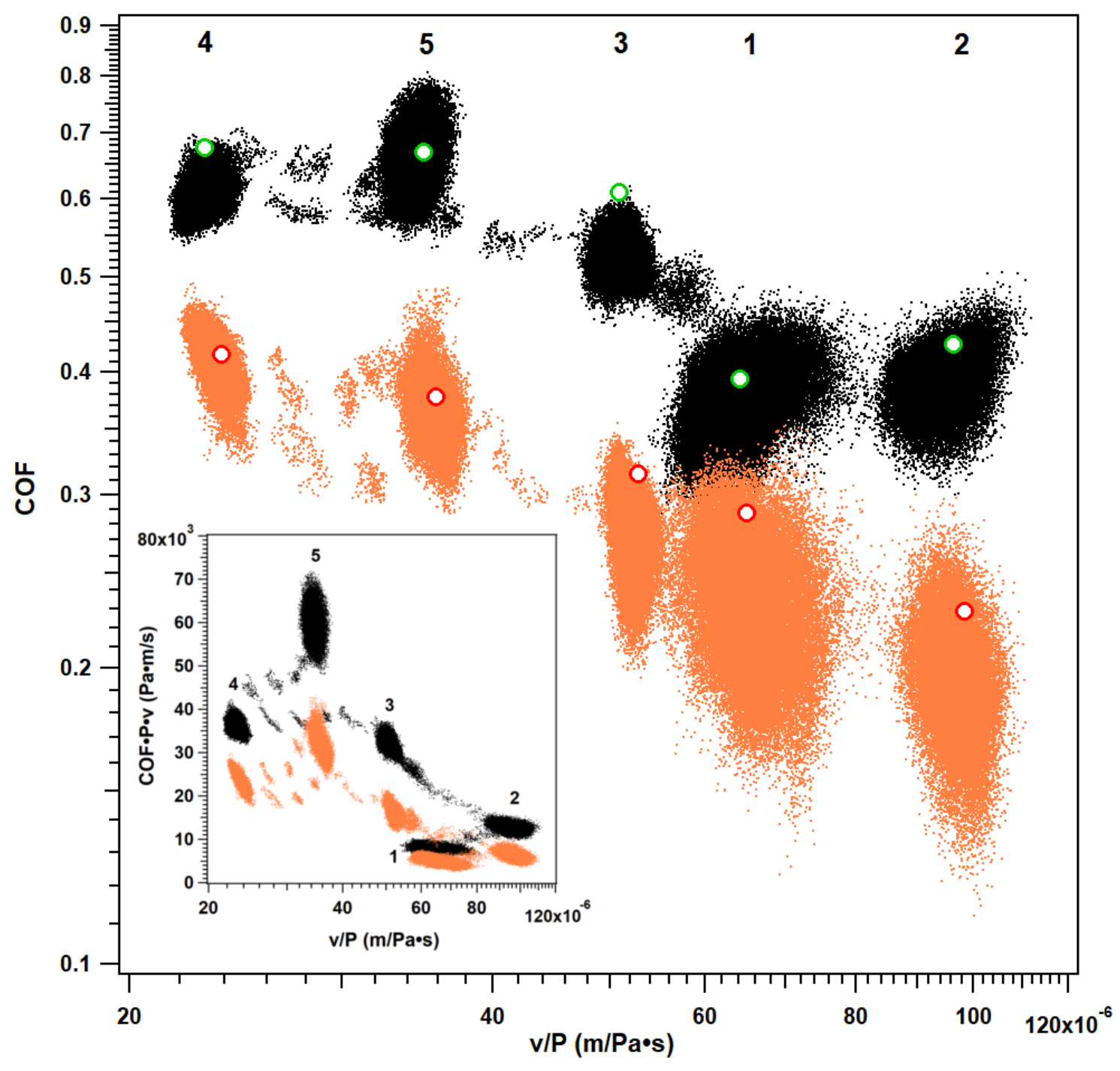

$178 \quad$ Fig 2. Stribeck (open circles) and Stribeck+ (clusters) curves corresponding to the PEEK ring using PL-4217 (black) and PL-7106 (orange). Numbers correspond to step numbers in Table 1. Inset: Implied ring wear rate as a function of pseudo-Sommerfeld number for PL-4217 (black) and PL-7106 (orange). 